\title{
Diversité de la macrofaune des sols cultivés sur les Hautes- Terres de Madagascar
}

\author{
Diane Rakotomanga ${ }^{(1)}$, Éric Blanchart ${ }^{(2)}$, Bodovololona Rabary ${ }^{(3)}$, \\ Richard Randriamanantsoa ${ }^{(3)}$, Malalatiana Razafindrakoto ${ }^{(2)}$, Patrice Autfray ${ }^{(4)}$ \\ (1) Bordeaux Sciences Agro. 1, Cours du Général de Gaulle. F-33175 Gradignan (France). E-mail : diane.rakotomanga@ \\ hotmail.fr \\ (2) IRD. UMR Éco\&Sols. Laboratoire des RadioIsotopes. BP 3383. 101 Antananarivo (Madagascar). \\ (3) FOFIFA. BP 230. 110 Antsirabe (Madagascar). \\ (4) CIRAD UR AIDA. BP 319. 110 Antsirabe (Madagascar).
}

Reçu le 1 février 2016, accepté le 29 aout 2016.

Description du sujet. L'article traite de la macrofaune du sol, composante clé du fonctionnement biologique du sol.

Objectifs. Cette étude, réalisée en 2015, a eu pour objectif de tester l'effet de pratiques culturales et de systèmes de culture sur la macrofaune du sol dans une zone d'agriculture-élevage des Hautes-Terres de Madagascar. Cinq systèmes de culture ont été étudiés : cultures annuelles labourées annuellement avec apport de fumier modéré (LAMF) ou élevé (LAEF), cultures fourragères pérennes sans labour et avec apport de fumier élevé (CFEF), cultures annuelles en agriculture de conservation sans labour et apport de fumier modéré (ACMF) et pâturages permanents, non labourés et sans apport de fumier (PP0F).

Méthode. Pour chaque système de culture, 12 parcelles d'expérimentation ou d'exploitation agricole ont fait l'objet d'un échantillonnage de la macrofaune du sol avec cinq monolithes de sol par parcelle, sur $30 \mathrm{~cm}$ de profondeur.

Résultats. Cinquante-deux espèces d'invertébrés, classées en 15 ordres taxonomiques et 6 groupes fonctionnels, ont été identifiées. Pour les cultures annuelles sans labour, l'absence de travail du sol a favorisé la diversité taxonomique et la densité de la macrofaune, n'a pas réduit la densité des vers blancs (larves de Coléoptères Scarabeoideae) rhizophages mais a entrainé une diminution des densités de vers de terre par rapport au mode de culture fourragère. En systèmes labourés, l'intensité d'application du fumier n'a eu d'effet ni sur les vers blancs ni sur les vers de terre.

Conclusions. Cette étude sur la macrofaune du sol dans cette région de Madagascar a permis de mieux percevoir les pratiques et les systèmes les plus favorables aux invertébrés utiles (comme les vers de terre) et plus défavorables aux invertébrés nuisibles (comme les vers blancs). Ceci permettra d'orienter les paysans dans la gestion de leurs terres.

Mots clés. Pratiques culturales, système de culture, fumier, faune du sol, Madagascar.

\section{Crop management and soil macrofauna diversity in the Highlands of Madagascar}

Description of the subject. This article deals with soil macrofauna as a key component of soil biological functioning.

Objectives. This original study, performed in 2015, aimed to compare the effect of contrasted land uses and cultural practices on soil macrofauna in a crop-livestock area in the Madagascar Highlands. Five cropping systems were investigated: annual crops under tillage with low (LAMF) and high (LAEF) manure inputs, permanent tilled forage crops with high manure inputs (CFEF), crops under conservation agriculture without tillage or permanent mulch cover and with medium manure inputs (ACMF), and permanent pastures without tillage and without manure (PPOF).

Method. In each of these five cropping systems, soil macrofauna was sampled in 12 plots from experimental trials and smallholder farms at the end of the rainy season. In each plot, soil macrofauna was hand-sorted from five soil monoliths at a $0-30 \mathrm{~cm}$ depth.

Results. Fifty-two species were identified and organized into 15 orders and six functional groups. In the annual cropping systems (ACMF), the absence of tillage and the presence of a permanent soil cover increased soil macrofauna diversity and density without reducing harmful white grubs (Scarabeoid beetle larvae), while earthworm density was reduced in comparison with the CFEF system. In conventional tillage cropping systems, manure input intensity (LAMF versus LAEF) had no specific effects on white grubs or earthworms.

Conclusions. This study on soil macrofauna in the Highlands of Madagascar helped to establish which agricultural practices and farming systems are favourable to beneficial soil invertebrates such as earthworms, and unfavourable to harmful insects 
such as white grubs. This will help farmers to achieve a more sustainable crop management.

Keywords. Cultivation, cropping system, farmyard manure, soil fauna, Madagascar.

\section{INTRODUCTION}

La diversité de la macrofaune des sols peut être utilisée à la fois comme outil de diagnostic de leur fertilité et comme ressource pour améliorer le fonctionnement du sol (Blanchart et al., 2006 ; Lavelle et al., 2006). De nombreuses espèces ou groupes taxonomiques d'invertébrés sont bénéfiques pour les sols. Les vers de terre, par exemple, en tant qu' «ingénieurs » du sol fournissent des services écosystémiques tels que la décomposition des matières organiques, le recyclage des nutriments et le maintien des propriétés physiques du sol favorables aux plantes (Lavelle et al., 1999). Cependant, cette biodiversité est menacée par l'intensification agricole et notamment par le travail répété du sol, l'absence de rotations culturales, l'absence d'apports organiques et l'utilisation abusive d'intrants de synthèse (Lavelle et al., 1999). Sur les Hautes-Terres de Madagascar, certains organismes de la macrofaune du sol sont aussi reconnus comme nuisibles par les agriculteurs; ces bioagresseurs des cultures sont notamment présents sur le riz pluvial, en pleine expansion dans cette région (Raboin et al., 2013 ; Sester et al., 2015). Les attaques de larves de Coléoptères Scarabeoideae (communément appelés vers blancs) représentent une contrainte majeure pour le riz (Avelino et al., 2012). La présente étude vise à étudier les interactions existant entre les pratiques culturales sur les Hautes-Terres de Madagascar et la macrofaune du sol, et de comprendre ainsi les déterminants de la diversité et de la densité de la macrofaune du sol dans les systèmes cultivés de Madagascar. Cette étude s'est concentrée sur une région agricole des Hautes-Terres caractérisée par des exploitations mixtes agricultureélevage laitier. Nous avons comparé des systèmes conventionnels avec d'autres systèmes basés sur des pâturages permanents extensifs ou des systèmes intensifs en agriculture de conservation. Les études sur la macrofaune du sol ont été réalisées en considérant :

- la macrofaune dans son ensemble,

- l'étude par groupes fonctionnels,

- avec une attention particulière sur les vers de terre (faune considérée comme bénéfique) et les vers blancs (faune essentiellement nuisible), à partir :

- d'une caractérisation des pratiques des agriculteurs dans 12 exploitations en se focalisant sur les apports de fumier, les cultures pratiquées, le travail du sol et la gestion des insectes ravageurs grâce aux traitements insecticides appliqués ;

- des prélèvements de macrofaune du sol dans des parcelles sélectionnées au sein de ces exploitations, complétés par des prélèvements sur pâturages et cultures annuelles conduites avec les principes de l'agriculture de conservation (Djigal et al., 2011) en situations expérimentales.

Selon des études réalisées à Madagascar et dans d'autres régions du monde en zone tropicale sur les relations entre pratiques et macrofaune du sol (Blanchart et al., 2006 ; Alvarez, 2012 ; Avelino et al., 2012 ; Bartz et al., 2013 ; Ratnadass et al., 2013), nous avons posé les hypothèses suivantes :

- la présence de fumier impacte positivement la diversité taxonomique, la densité et la biomasse de la macrofaune du sol. Le fumier favorise la présence et l'activité des vers de terre, mais aussi celui des vers blancs rhizophages nuisibles des cultures;

- l'absence de labour combinée à une activité racinaire des plantes pérennes favorise la présence de la macrofaune du sol ;

- l'utilisation de cultures annuelles comme le riz et le maïs favorise la présence de vers blancs rhizophages des cultures ;

- le recours aux traitements insecticides répétés pour lutter contre les vers blancs impacte l'ensemble de la macrofaune du sol.

\section{MATÉRIEL ET MÉTHODES}

\subsection{Caractérisation des sites d'études}

Douze exploitations en agriculture-élevage laitier, représentatives de la région Vakinankaratra (Alvarez, 2012), ont été sélectionnées à partir d'un réseau d'exploitations étudié dans le cadre du projet BIOVA (Recyclage des masses végétales et animales dans les systèmes d'agriculture-élevage; https://www. biova-project.org). Ces exploitations sont concentrées autour de la ville d'Antsirabe (20 $\mathrm{km}^{2}$ environ) dans un souci de limiter la variabilité du milieu physique (sols, précipitations, altitude) (Figure 1). Leur taille et celle des troupeaux sont deux critères importants de différenciation. Ces caractéristiques déterminent en particulier les quantités de fumier disponibles à l'échelle de l'exploitation. À ces exploitations ont été associés des dispositifs expérimentaux proches de la commune rurale d'Andranomanelatra. Au final, au sein de cette région, considérée comme climatiquement homogène, 36 parcelles paysannes (de superficie moyenne de $2735 \mathrm{~m}^{2}$ ) ont été choisies dont 24 parcelles en cultures annuelles diversifiées et 12 parcelles de 


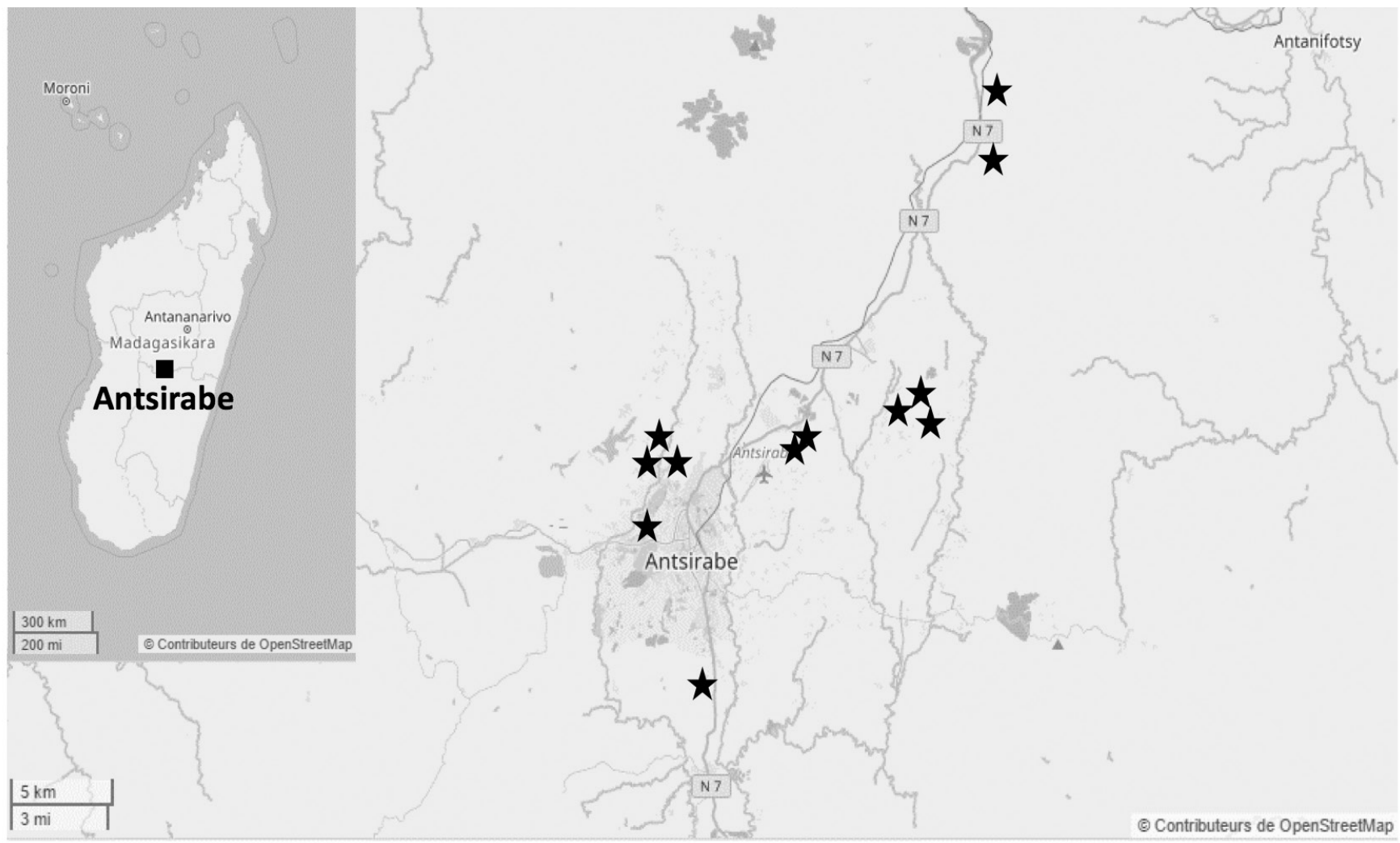

Figure 1. Localisation des douze exploitations de la région d'Antsirabe ayant servi pour l'étude - Map presenting the location of the twelve farms studied in Antsirabe area.

cultures fourragères. Sur des sites expérimentaux voisins de ces exploitations, 12 parcelles de rotation avec des cultures annuelles (de superficie moyenne de $76 \mathrm{~m}^{2}$ ) et 12 parcelles en pâturage permanent (de superficie moyenne de $1459 \mathrm{~m}^{2}$ ) ont été sélectionnées. L'altitude des parcelles se situe entre 1472 à $1682 \mathrm{~m}$ et la pluviométrie moyenne était de $1358 \mathrm{~mm}$ pour la période 2002 à 2014, avec pour la saison culturale de septembre 2014 à aout 2015, un total de $1563 \mathrm{~mm}$. La latitude des parcelles variait entre $19688^{\circ}$ et $19911^{\circ}$ Sud et leur longitude de $47007^{\circ}$ à $47168^{\circ}$ Est. Dans cette région, les sols appartiennent aux séries volcano-sédimentaires avec une texture argileuse (teneur en argiles $>50 \%$ ) (Dusserre et al., 2012). Les caractéristiques du sol en surface $(0-10 \mathrm{~cm})$ varient de 32,2 à $46,7 \%$ pour l'humidité gravimétrique, de 4,4 à 5,0 pour l'acidité ( $\mathrm{pH}$ eau 1/2,5 m/V), de 37,3 à 49,4 g. $\mathrm{kg}^{-1}$ pour le carbone organique (Walkley \& Black) et 4,9 à $11,9 \mathrm{mg} \cdot \mathrm{kg}^{-1}$ pour le phosphore assimilable (méthode Olsen, extraction par une solution de bicarbonate de sodium $0,5 \mathrm{M}$ à $\mathrm{pH} 8,5)$.

\subsection{Caractérisation des pratiques agricoles des exploitations et des sites expérimentaux}

La caractérisation des exploitations a permis de constater que celles-ci possédaient toutes des parcelles de bas-fonds, des parcelles de plantes fourragères et des parcelles de cultures vivrières sur «tanety» (sommet ou versant des collines en langue malgache) ainsi qu'une étable où sont placés les vaches laitières et les bœufs de trait. Les flux de biomasse concernaient principalement les flux de fumier et de récoltes. Certaines exploitations, quelle que soit leur taille, étaient autosuffisantes en biomasse puisqu'elles n'ont importé ni paille ni foin de l'extérieur. Cependant, la décision d'apporter plus de fumier à certaines parcelles se fait selon les objectifs de production de chaque exploitation, la stratégie et la disponibilité du fumier au sein de l'exploitation. Pour l'ensemble de ces exploitations, le fumier produit annuellement était appliqué au niveau des «tanety», principalement en début de saison de culture (octobre-novembre). Nous avons déterminé les différents systèmes de culture pratiqués et leur gestion actuelle par des agriculteurs avec une considération portant sur :

- les flux de biomasse et les apports de fumier,

- la fréquence de travail du sol,

- l'utilisation de pesticides.

Trois modes contrastés de gestion des cultures au sein de ces exploitations ont été identifiés pour chaque exploitation, selon les cultures, la quantité de fumier et le labour, présentés dans le tableau 1 : 
498 Biotechnol. Agron. Soc. Environ. 2016 20(4), 495-507

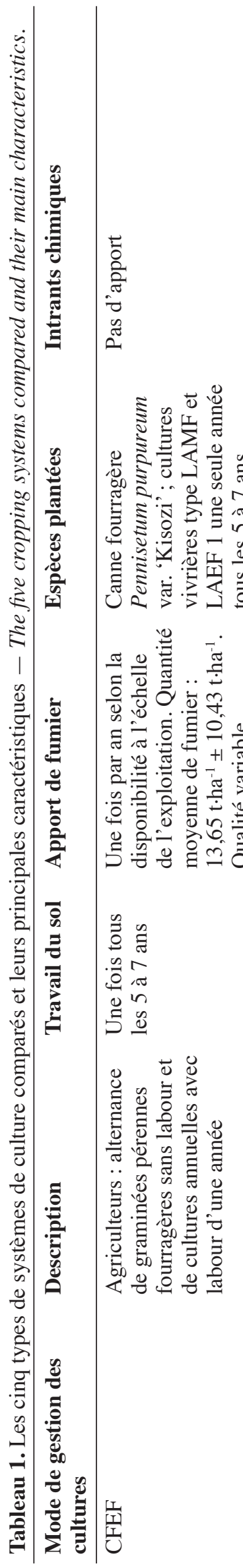

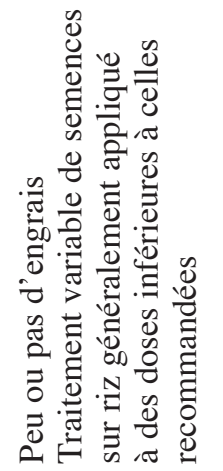

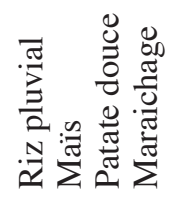

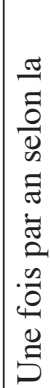
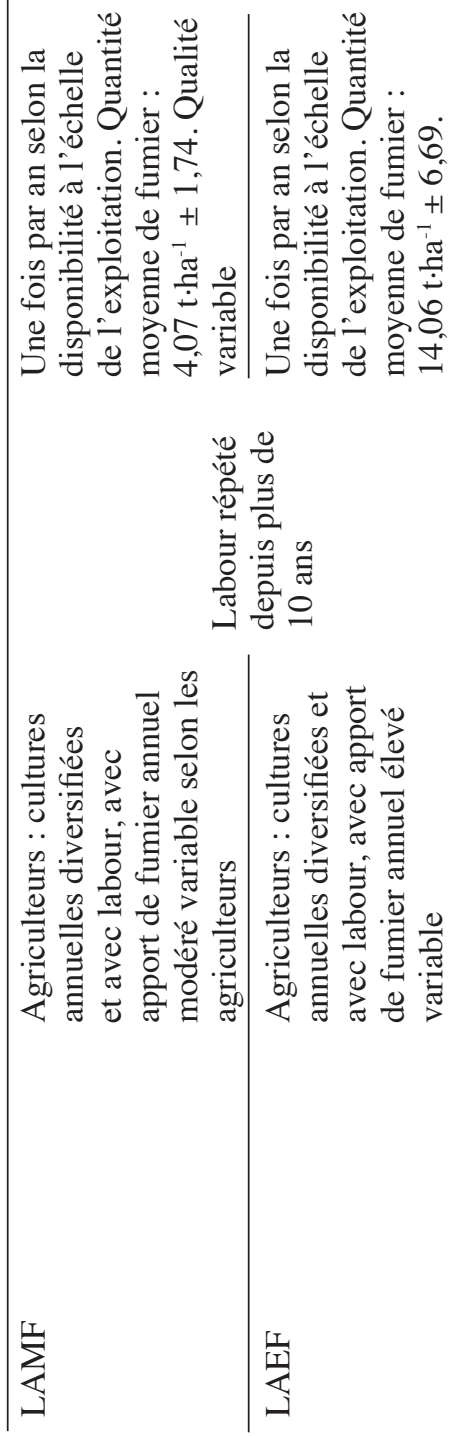

Rakotomanga D., Blanchart É., Rabary B. et al.

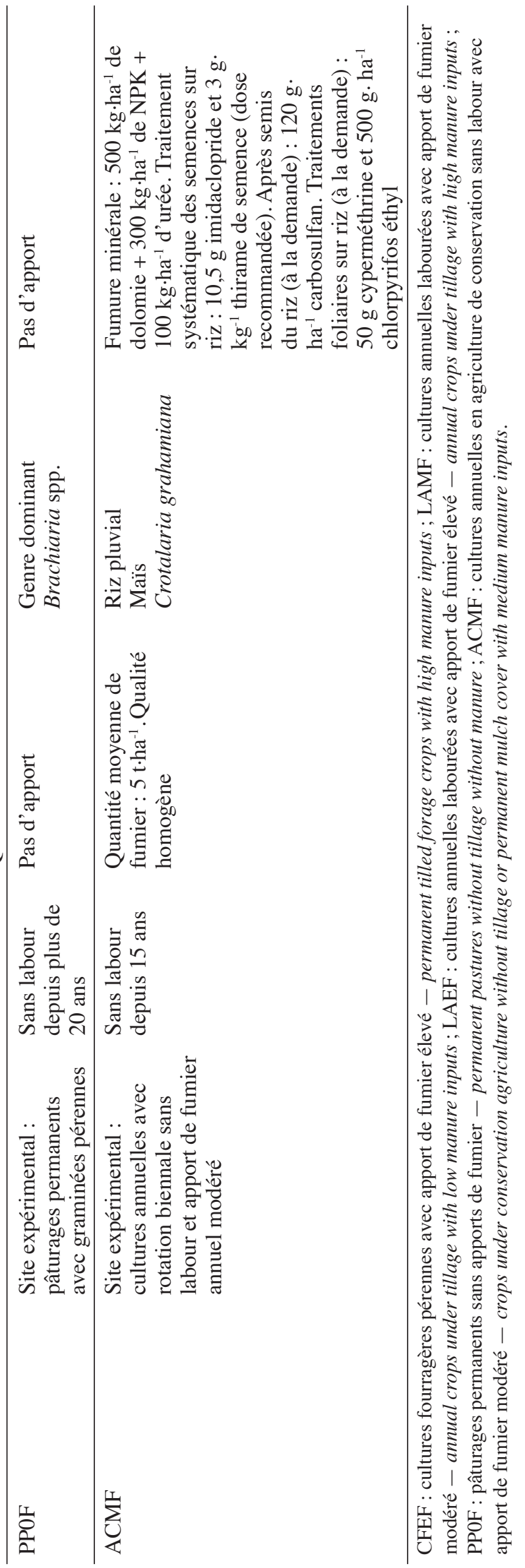


- parcelles avec cultures pérennes composées de canne fourragère (Pennisetum purpureum var. 'Kisozi') labourées seulement une fois tous les cinq à sept ans et fertilisées sur cette dernière campagne agricole avec 13,7 that ${ }^{-1}$ en moyenne de fumier (Système Mixte, CFEF).

- parcelles avec cultures annuelles diversifiées, principalement riz, maïs, patate douce, maraichage, labourées régulièrement et fertilisées soit avec un apport modéré de fumier estimé en moyenne à $4,1 \mathrm{t}$. ha $^{-1}$ environ (Labour et Apport Modéré de Fumier, LAMF), soit avec un apport élevé de fumier estimé en moyenne à 14,1 th.ha ${ }^{-1}$ environ (Labour et Apport Élevé de Fumier, LAEF); un seul traitement insecticide de synthèse est appliqué au niveau des semences de riz et de maiis et généralement inférieur aux doses recommandées.

La définition de ces modes de gestion des cultures a permis de sélectionner dans chaque exploitation les parcelles où ont eu lieu les prélèvements de la macrofaune: une parcelle prélevée pour le mode de gestion CFEF et deux autres dans les modes de gestion LAMF et LAEF quand ceux-ci étaient discernables au sein de l'exploitation. Dans le cas contraire, nous avons sélectionné soit LAMF, soit LAEF. Au sein de chaque exploitation, les agriculteurs nous ont assuré que ces parcelles étaient gérées de la sorte depuis au moins 10 années. Deux autres modes contrastés de gestion des sols et des cultures ont été sélectionnés pour servir de références au niveau de sites expérimentaux (Tableau 1) :

- des pâturages permanents (PPOF), composés de plantes pérennes du genre Brachiaria, qui n'ont pas été labourés depuis plus de 10 ans et qui ne reçoivent aucun apport de fumier ;

- des parcelles de cultures annuelles en rotation biannuelle riz et maïs associés avec Crotalaria grahamiana, légumineuse de couverture, gérées selon les principes de l'agriculture de conservation (ACMF) pendant 15 années : absence de travail du sol, couverture permanente et utilisation de rotations et d'associations culturales; elles reçoivent $5 \mathrm{t} \cdot \mathrm{ha}^{-1}$ de fumier par an et régulièrement des applications d'insecticides comprenant un traitement systématique des semences aux doses recommandées et selon les années en fonction des traitements de sol (carbosulfan) ou foliaires (cyperméthrine et chlorpyrifos éthyl).

Ces cinq modes de gestion des cultures ont ensuite été comparés entre eux comme suit.

Pour les cultures annuelles :

- comparaison de ACMF et LAMF : les quantités de fumier apportées étant sensiblement les mêmes, on peut étudier l'effet du labour, de l'impact des pesticides et de la diversification culturale.
- comparaison de LAMF et LAEF : le labour y étant effectué tous les ans, on peut étudier précisément l'impact de la quantité de fumier épandue.

Pour les cultures pérennes, on a comparé PPOF et CFEF, les parcelles de PPOF n'ayant pas été labourées depuis au moins dix ans et celles de CFEF depuis quatre à cinq ans ; on a également pu étudier les effets de la quantité de fumier épandue ainsi que ceux de la fréquence du labour.

\subsection{Prélèvement et caractérisation de la macrofaune}

Des échantillonnages de macrofaune (macro-invertébrés visibles à l'œil nu) du sol ont été réalisés dans les parcelles d'expérimentation et les parcelles paysannes, soit au total sur 60 parcelles considérées comme indépendantes les unes des autres, en prenant 12 parcelles par système de culture. La variabilité biophysique est considérée comme limitée et les prélèvements ont été effectués en fin de saison des pluies (mars-avril) sur des sols humides sur une période de 30 jours. La méthode de prélèvement Tropical Soil Biology and Fertility (TSBF) (Anderson et al., 1993) a été utilisée mais modifiée pour être moins destructrice. Des échantillons de sol, «monolithes » de $25 \mathrm{~cm}$ de côté sur une profondeur de $30 \mathrm{~cm}$, ont été prélevés puis coupés en trois horizons de profondeur $0-10 \mathrm{~cm}, 10-20 \mathrm{~cm}$ et $20-30 \mathrm{~cm}$. Dans chaque parcelle, cinq monolithes ont été répartis le long des diagonales selon une croix. Les premiers trous sont décalés d'au moins $1 \mathrm{~m}$ des bords de la parcelle, quelle que soit la taille de celle-ci. La macrofaune du sol a été triée à la main sur le terrain et conservée dans des flacons en plastique de $30 \mathrm{ml}$ remplis d'alcool à $70^{\circ} \mathrm{C}$. L'identification a été réalisée au laboratoire à l'aide d'une loupe binoculaire en utilisant des clés de détermination des arthropodes, des insectes ainsi que des vers blancs (Dechambre, 1986 ; Lacroix, 1989 ; Lacroix, 1993 ; Lacroix, 1997 ; Lacroix, 1998 ; Randriamanantsoa et al., 2010). Razafindrakoto et al. (2010) et Csuzdi et al. (2012) ont servi pour l'identification des vers de terre. La densité de macrofaune est exprimée en nombre d'individus par $\mathrm{m}^{2}$. La richesse taxonomique est traduite par le nombre d'ordres obtenus. La macrofaune a été classée en ordres puis en groupes fonctionnels définis par Turbé et al. (2010) et Ratnadass et al. (2013) pour mieux appréhender l'impact des différents modes de gestion des cultures sur la macrofaune du sol :

- les coprophages se nourrissent des bouses et les enfouissent au fond des galeries pour servir de nourriture à leurs larves,

- les saprophages se nourrissent de litières, de racines mortes et de bois en décomposition,

- les géophages se nourrissent de la matière organique contenue dans le sol, 
- les phytophages consomment les organes aériens des plantes,

- les prédateurs se nourrissent d'autres organismes du sol,

- les rhizophages consomment des racines vivantes.

\subsection{Analyse des données}

Les résultats des enquêtes et les mesures collectées sur le terrain ont été recoupées pour définir les principaux modes de gestion des cultures à partir des caractéristiques de l'exploitation, de la production de fumier et du plan de fumure. Les données collectées à l'aide de GPS ont été traitées avec le logiciel QGIS
2.8.1 de manière à réaliser des cartes d'exploitations avec la mesure de la superficie des parcelles et des flux de biomasse. Cela a permis de définir dans un premier temps les principaux systèmes de culture et les quantités d'intrants appliqués. La figure $\mathbf{2}$ montre la démarche de traitement de l'information qui a été validée dans un deuxième temps avec les agriculteurs en recoupant les données parcelles (quantité de fumier appliquée) et les données exploitation (quantité totale de fumier produite). Cette méthode s'est inspirée d'autres études réalisées avec des exploitations familiales (Tonneau et al., 2002). La densité a été calculée pour chaque groupe fonctionnel recensé au sein de chaque parcelle sur Excel ${ }^{\circledR}$ en faisant la moyenne des résultats des

Récolte des données au GPS avec calcul de la superficie de chaque parcelle pour chaque exploitation

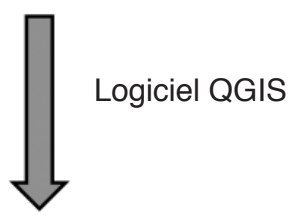

Élaboration d'une carte de chaque exploitation localisant chaque parcelle et mettant en évidence les quantités de fumier apportées
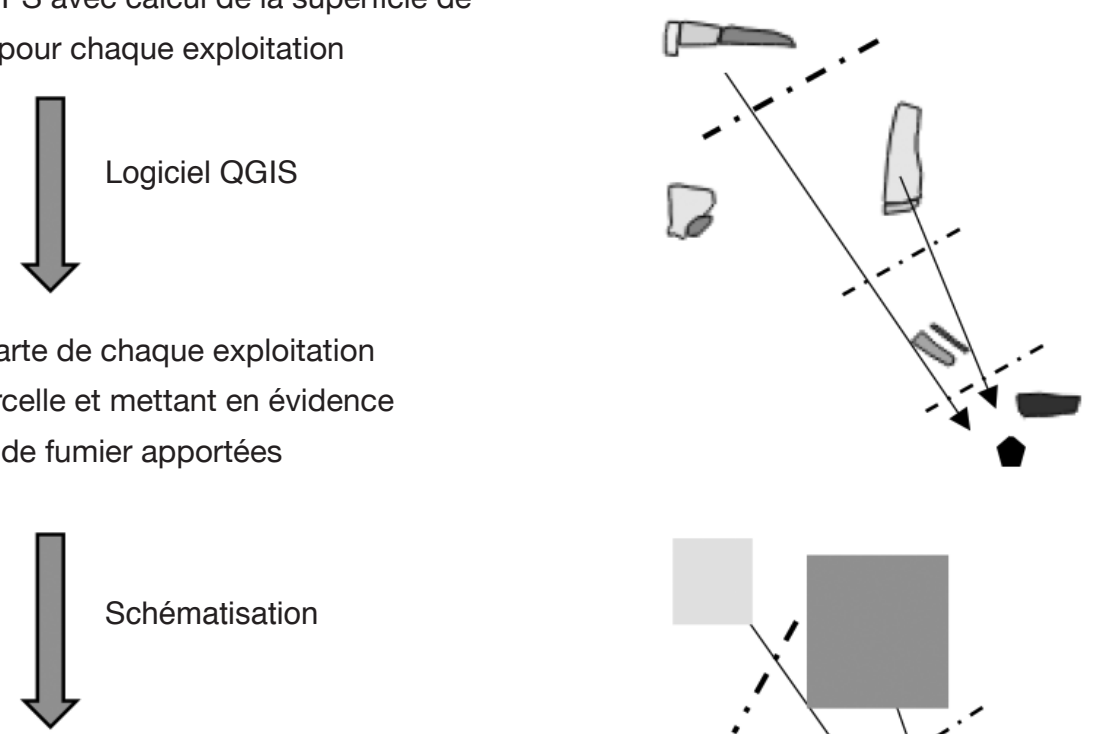
systèmes de culture principaux
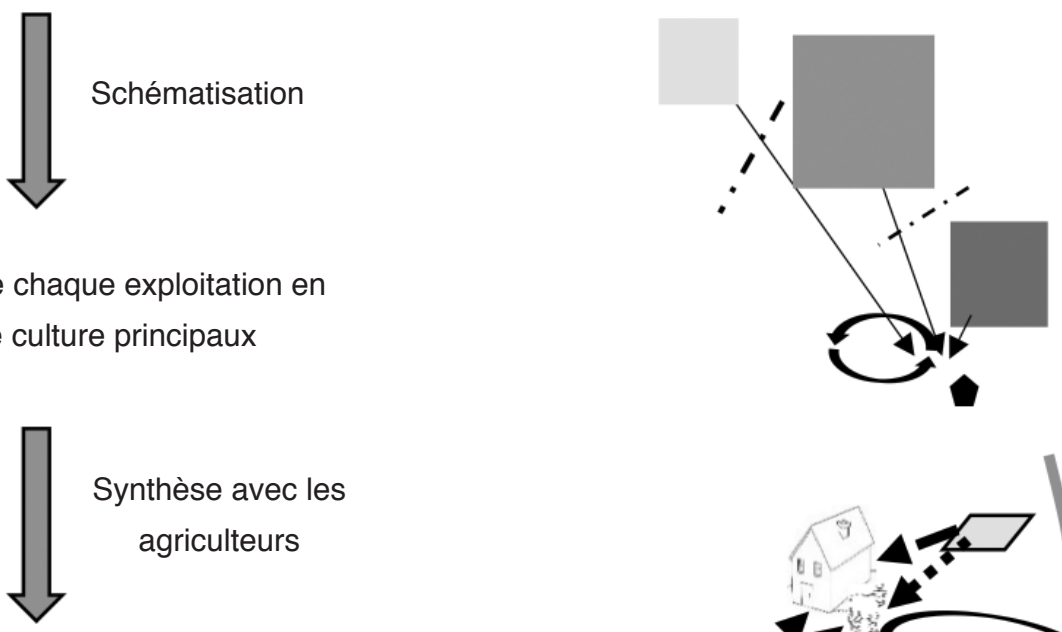

\section{Recoupement des données obtenues par système de} culture à l'échelle de l'exploitation agricole

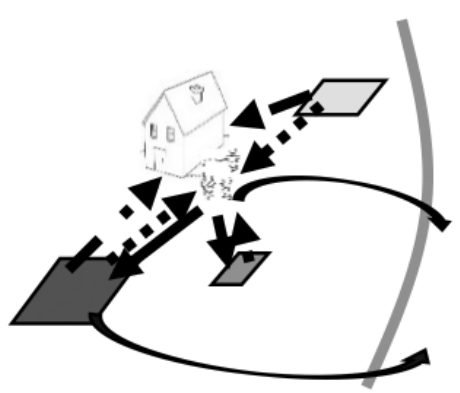

Figure 2. Démarche en quatre étapes pour la caractérisation des systèmes de culture à l'échelle de l'exploitation agricole et le choix des parcelles d'étude - Protocol elaborated at the farm level for cropping systems characterization and studied plot selection.

La couleur du bloc de culture représente la quantité de fumier épandue : plus elle est foncée, plus il y a de fumier, sa taille étant proportionnelle à sa superficie réelle - The square color represents the manure input quantity: the darker it is, higher is the manure quantity with the height being proportional to the real area. 
cinq monolithes pour chaque parcelle. Pour toutes les analyses statistiques, on a utilisé le logiciel XLSTAT ${ }^{\circledR}$ 2015 (Version 2015.1.01). Les comparaisons des densités moyennes de macrofaune pour chaque mode de gestion ont été faites pour les différents groupes fonctionnels avec un nombre identique de 12 répétitions par système de culture. Le test statistique a été réalisé après une transformation de type racine carrée, suivi d'un test de Fisher ou d'un test de Kruskal-Wallis et Mann-Whitney en cas de non-normalité des données, avec un seuil de signification de $5 \%$.

\section{RÉSULTATS}

\subsection{Densité de la macrofaune par ordre taxonomique et par profondeur de sol}

Cinquante-deux espèces ont été identifiées à partir des 5009 individus collectés durant cet échantillonnage ; ils ont été répartis en 15 ordres différents et dans les six groupes fonctionnels (Tableau 2). En termes de répartition selon la profondeur pour les cultures annuelles, la densité de la macrofaune est significativement plus importante dans l'horizon 0-10 $\mathrm{cm}$ avec ACMF, alors qu' elle parait mieux répartie sur les trois profondeurs pour LAMF et LAEF, tandis que cette différence n'est pas significative entre les parcelles sur 0-30 cm (Tableau 3). Toujours pour les cultures annuelles, la macrofaune est plus abondante et plus diversifiée dans le mode de gestion en ACMF, où elle est représentée par un plus grand nombre d'ordres taxonomiques (15 groupes taxonomiques pour ACMF contre 12 pour LAMF et 13 pour LAEF) malgré l'application continue de traitements insecticides (Tableau 3). Les densités de macrofaune observées entre les modes LAMF et LAEF ne sont pas significativement différentes, suggérant que les apports différenciés en quantité de fumier n'ont pas un effet particulier sur la macrofaune. Pour les cultures pérennes, aucune différence significative de la densité de la macrofaune n'apparait pour chaque horizon étudié séparément, alors qu'elle est significative sur 0-30 cm (Tableau 3). La densité de la macrofaune sur 0-30 $\mathrm{cm}$ pour CFEF est de $74 \%$ supérieure par rapport à PP0F. Par contre, une plus grande richesse taxonomique en ordres et en espèces a été constatée au niveau des pâturages permanents (PPOF). Ces pâturages présentaient une plus forte proportion de Coléoptères et une plus faible proportion de vers de terre (Oligochètes), en comparaison des parcelles gérées en CFEF. En considérant l'ensemble de la profondeur $0-30 \mathrm{~cm}$ et les cinq systèmes de culture, il s'avère que certains ordres sont plus discriminants que d'autres. La densité des groupes de macrofaune suivants montre bien la différence entre chaque mode de gestion : il s'agit des Arachnides, des Chilopodes, des Coléoptères sans les vers blancs, des Dermaptères, des Oligochètes et des Orthoptères; ces groupes peuvent ainsi être utilisés pour discriminer les systèmes de culture.

Tableau 2. Groupes taxonomiques identifiés lors des prélèvements autour d'Antsirabe au cours de la campagne 20142015 - Taxonomic groups identified around Antsirabe city during the 2014-2015 cropping period.

\begin{tabular}{|c|c|c|c|c|c|}
\hline Phylum & Classe & Ordre & $\begin{array}{l}\text { Super-famille, } \\
\text { famille }\end{array}$ & Espèce ou nom commun & $\begin{array}{l}\text { Groupe } \\
\text { fonctionnel }^{1}\end{array}$ \\
\hline \multirow[t]{6}{*}{ Annelida } & Oligochaeta & Haplotaxidae & Acanthodrilidae & $\begin{array}{l}\text { Dichogaster affinis } \\
\text { Dichogaster bolaui } \\
\text { Dichogaster saliens }\end{array}$ & $\begin{array}{l}\text { Saprophage } \\
\text { Saprophage } \\
\text { Saprophage }\end{array}$ \\
\hline & & & Enchytreidae & Enchytréide & Saprophage \\
\hline & & & Glososcolecidae & Pontoscolex corethrurus & Géophage \\
\hline & & & Kynotidae & Kynotus parvus & Géophage \\
\hline & & & Megascolecidae & $\begin{array}{l}\text { Amynthas sp. } \\
\text { Amynthas affinis } \\
\text { Amynthas corticis } \\
\text { Amynthas minimus }\end{array}$ & $\begin{array}{l}\text { Saprophage } \\
\text { Saprophage } \\
\text { Saprophage } \\
\text { Saprophage }\end{array}$ \\
\hline & & & Moniligastridae & Drawida barweli & Saprophage \\
\hline Annelida & Oligochaeta & Haplotaxidae & & Cocon & Non fonctionnel \\
\hline Arthropoda & Arachnida & Araneae & Aranae & Sp. & Prédateur \\
\hline Arthropoda & Myriapoda & Diplopoda & Iulidae & Sp. & Phytophage \\
\hline Arthropoda & Myriapoda & Chilopoda & $\begin{array}{l}\text { Lithobiidae } \\
\text { Scolopendridae }\end{array}$ & $\begin{array}{l}\text { Sp. } \\
\text { Sp. }\end{array}$ & $\begin{array}{l}\text { Prédateur } \\
\text { Prédateur }\end{array}$ \\
\hline
\end{tabular}


Tableau 2 (suite). Groupes taxonomiques identifiés lors des prélèvements autour d'Antsirabe au cours de la campagne 2014-2015 - Taxonomic groups identified around Antsirabe city during the 2014-2015 cropping period.

\begin{tabular}{|c|c|c|c|c|c|}
\hline Phylum & Classe & Ordre & $\begin{array}{l}\text { Super-famille, } \\
\text { famille }\end{array}$ & Espèce ou nom commun & $\begin{array}{l}\text { Groupe } \\
\text { fonctionnel }^{1}\end{array}$ \\
\hline Arthropoda & Myriapoda & Chilopoda & Geophilidae & $\begin{array}{l}\text { Symphyle } \\
\text { Sp. }\end{array}$ & $\begin{array}{l}\text { Prédateur } \\
\text { Prédateur }\end{array}$ \\
\hline Arthropoda & Insecta & Coleoptera & $\begin{array}{l}\text { Brachinidae } \\
\text { Carabidae } \\
\text { Cetonidae } \\
\text { Cryptophagidae } \\
\text { Dynastidae } \\
\\
\text { Elateridae } \\
\text { Elateridae } \\
\text { Eucnemidae } \\
\text { Hopliidae } \\
\text { Melolonthidae } \\
\\
\text { Orphinidae } \\
\text { Pselaphidae } \\
\text { Scarabeoidea } \\
\text { Sericidae } \\
\text { Staphylinidae } \\
\text { Staphylinidae }\end{array}$ & $\begin{array}{l}\text { Metabrachinus connectoides } \\
\text { Polycleus } \\
\text { Sp. } \\
\text { Celidota parvula } \\
\text { Euryomia argentea } \\
\text { Cryptophage } \\
\text { Heteroconus paradoxus } \\
\text { Heteronychus plebeius } \\
\text { Hexodon unicolor } \\
\text { Taupin } \\
\text { Sp. } \\
\text { Gonocephalum } \\
\text { Paramorphochelus cornutus } \\
\text { Enaria melanictera } \\
\text { Hoplochelus marginalis } \\
\text { Apicencya waterloti } \\
\text { Triodontus nitidulus } \\
\text { Sp. } \\
\text { Sp. } \\
\text { Larve de Serica } \\
\text { Staphylin } \\
\text { Nymphe de Staphylin }\end{array}$ & $\begin{array}{l}\text { Phytophage } \\
\text { Rhizophage } \\
\text { Prédateur } \\
\text { Coprophage } \\
\text { Coprophage } \\
\text { Phytophage } \\
\text { Phytophage } \\
\text { Rhizophage } \\
\text { Saprophage } \\
\text { Rhizophage } \\
\text { Rhizophage } \\
\text { Phytophage } \\
\text { Rhizophage } \\
\text { Rhizophage } \\
\text { Rhizophage } \\
\text { Rhizophage } \\
\text { Rhizophage } \\
\text { Prédateur } \\
\text { Prédateur } \\
\text { Indéterminé } \\
\text { Prédateur } \\
\text { Non fonctionnel }\end{array}$ \\
\hline Arthropoda & Insecta & Dermaptera & Pigydicranidae & Euborelia stati & Prédateur \\
\hline Arthropoda & Insecta & Dictyoptera & Blattidae & Blatte & Saprophage \\
\hline Arthropoda & Insecta & Diptera & Tipulidae & Larve de tipule & Saprophage \\
\hline Arthropoda & Insecta & Homoptera & Pentatomoidae & Punaise & Phytophage \\
\hline Arthropoda & Insecta & Hymenoptera & Formicidae & $\begin{array}{l}\text { Camponotus } \\
\text { Monomorium } \\
\text { Nylanderia } \\
\text { Paraparatrechina } \\
\text { Tetramorium }\end{array}$ & $\begin{array}{l}\text { Saprophage } \\
\text { Saprophage } \\
\text { Saprophage } \\
\text { Saprophage } \\
\text { Saprophage }\end{array}$ \\
\hline Arthropoda & Insecta & Isoptera & Termitidae & Termite & Saprophage \\
\hline Arthropoda & Insecta & Lepidoptera & & Chenille & Phytophage \\
\hline Arthropoda & Insecta & Orthoptera & $\begin{array}{l}\text { Acrididae } \\
\text { Gryllidae } \\
\text { Gryllotalpidae }\end{array}$ & $\begin{array}{l}\text { Locusta migratoria } \\
\text { Grillon } \\
\text { Gryllotalpa sp. }\end{array}$ & $\begin{array}{l}\text { Phytophage } \\
\text { Phytophage } \\
\text { Phytophage }\end{array}$ \\
\hline Arthropoda & Insecta & Thysanoura & Lepismatidae & Lépisme & Prédateur \\
\hline Arthropoda & Malacostracae & Isopoda & Agnaridae & Sp. & Saprophage \\
\hline
\end{tabular}

${ }^{1}$ : d'après Ratnadass et al., 2013 - according to Ratnadass et al., 2013. 


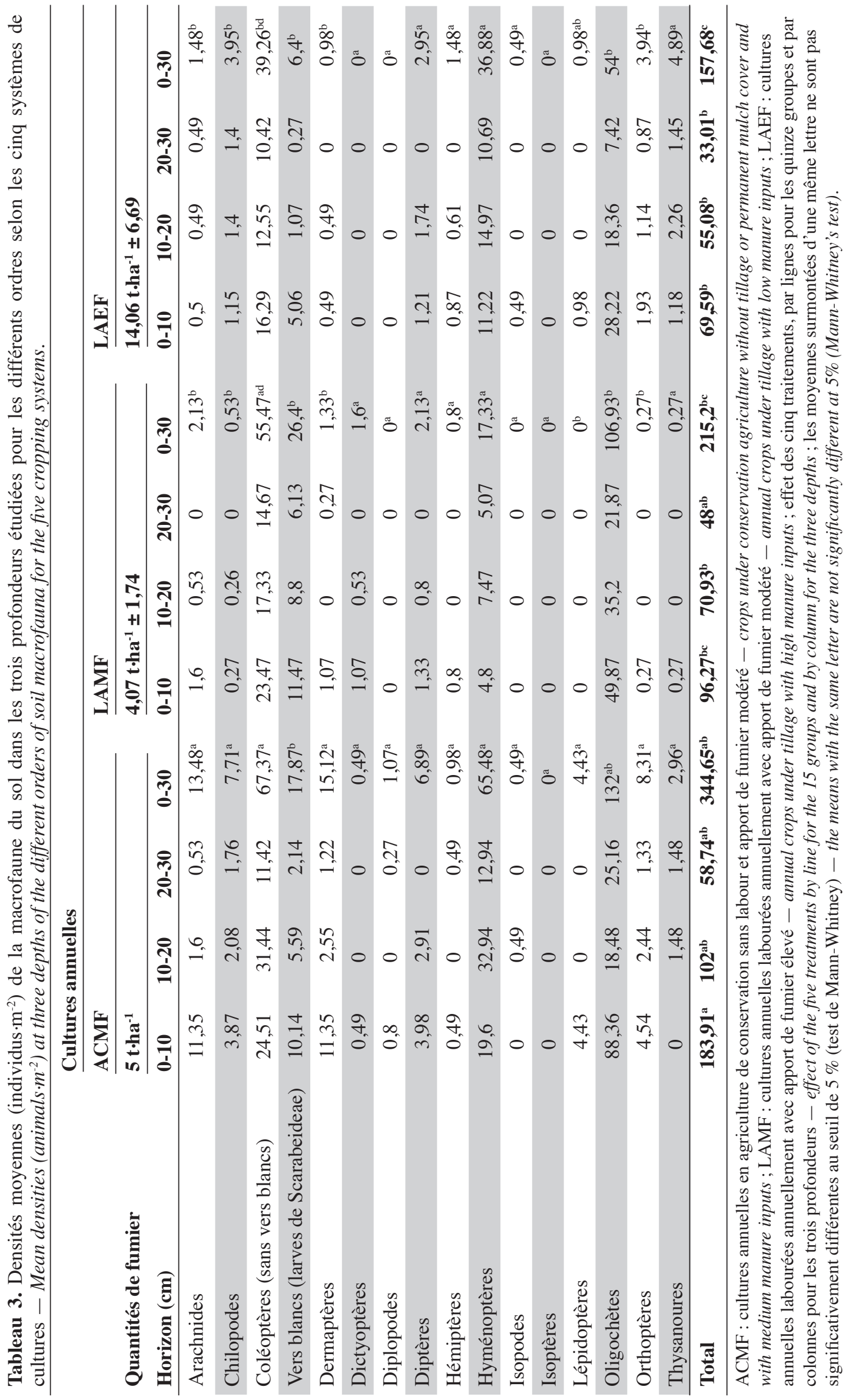


Tableau 3 (suite). Densités moyennes (individus $\cdot \mathrm{m}^{-2}$ ) de la macrofaune du sol dans les trois profondeurs étudiées pour les différents ordres selon les cinq systèmes de cultures - Mean densities (animals $\cdot \mathrm{m}^{-2}$ ) at three depths of the different orders of soil macrofauna for the five cropping systems.

\begin{tabular}{|c|c|c|c|c|c|c|c|c|}
\hline \multirow{4}{*}{$\begin{array}{l}\text { Quantités de fumier } \\
\text { Horizon }(\mathbf{c m})\end{array}$} & \multicolumn{8}{|c|}{ Cultures pérennes } \\
\hline & \multicolumn{4}{|l|}{ PP0F } & \multicolumn{4}{|l|}{ CFEF } \\
\hline & \multicolumn{4}{|l|}{0 t.ha ${ }^{-1}$} & \multicolumn{4}{|c|}{$13,65 \mathrm{t}^{\prime} \mathrm{ha}^{-1} \pm 10,43$} \\
\hline & $0-10$ & $10-20$ & $20-30$ & 0-30 & $0-10$ & $10-20$ & $20-30$ & 0-30 \\
\hline Arachnides & 1,33 & 1,07 & 0,53 & $2,93^{\mathrm{ab}}$ & 1,6 & 0,53 & 0,27 & $2,4^{\mathrm{b}}$ \\
\hline Chilopodes & 1,87 & 1,07 & 0,8 & $3,74^{\mathrm{a}}$ & 0 & 0 & 0,27 & $0,27^{\mathrm{b}}$ \\
\hline Coléoptères sans vers blancs & 13,6 & 10,93 & 19,73 & $44,27^{\mathrm{ac}}$ & 13,33 & 11,47 & 8,27 & $33,07^{\mathrm{d}}$ \\
\hline Vers blancs (larves de coléoptères) & 20,53 & 15,73 & 9,6 & $45,87^{\mathrm{a}}$ & 14,4 & 9,6 & 4,27 & $28,27^{\mathrm{b}}$ \\
\hline Dermaptères & 0,27 & 0,27 & 0 & $0,53^{\mathrm{b}}$ & 1,07 & 0 & 0 & $1,07^{\mathrm{b}}$ \\
\hline Dictyoptères & 0 & 0,27 & 0 & $0,27^{\mathrm{a}}$ & 1,33 & 0,27 & 0 & $1,6^{\mathrm{a}}$ \\
\hline Diplopodes & 1,07 & 0,8 & 0 & $1,87^{\mathrm{a}}$ & 0,27 & 0 & 0 & $0,27^{\mathrm{a}}$ \\
\hline Diptères & 2,4 & 0,53 & 0 & $2,93^{\mathrm{a}}$ & 1,07 & 0,27 & 0,53 & $1,87^{\mathrm{a}}$ \\
\hline Hémiptères & 1,6 & 0,8 & 0 & $2,4^{\mathrm{a}}$ & 1,6 & 0,53 & 0,53 & $2,67^{\mathrm{a}}$ \\
\hline Hyménoptères & 9,87 & 8 & 2,13 & $20^{\mathrm{a}}$ & 8,53 & 19,73 & 6,67 & $34,93^{\mathrm{a}}$ \\
\hline Isopodes & 0 & 0 & 0 & $0^{\mathrm{a}}$ & 0 & 0 & 0 & $0^{\mathrm{a}}$ \\
\hline Isoptères & 6,4 & 4,53 & 0 & $10,93^{\mathrm{a}}$ & 0 & 0 & 0 & $0^{\mathrm{a}}$ \\
\hline Lépidoptères & 0 & 0 & 0 & $0^{\mathrm{b}}$ & 0,27 & 0 & 0 & $0,27^{\mathrm{ab}}$ \\
\hline Oligochètes & 61,07 & 19,73 & 7,73 & $88,53^{b}$ & 109,6 & 115,2 & 63,2 & $288^{\mathrm{a}}$ \\
\hline Orthoptères & 1,6 & 0,53 & 0,27 & $2,4^{\mathrm{b}}$ & 1,33 & 0,27 & 0 & $1,6^{\mathrm{bc}}$ \\
\hline Thysanoures & 1,07 & 0,53 & 0 & $1,6^{\mathrm{a}}$ & 0 & 0,8 & 0,8 & $1,6^{\mathrm{a}}$ \\
\hline Total & $122,67^{\text {ac }}$ & $64,8^{\mathrm{ab}}$ & $40,8^{\mathrm{ab}}$ & $228,27^{\mathrm{bc}}$ & $154,4^{\text {ac }}$ & $158,67^{\mathrm{a}}$ & $84,8^{\mathrm{a}}$ & $397,87^{a}$ \\
\hline
\end{tabular}

PPOF : pâturages permanents, non labourés et sans apport de fumier - permanent pastures without tillage and without manure ; CFEF : cultures fourragères pérennes sans labour et avec apport de fumier élevé - permanent tilled forage crops with high manure inputs ; effet des cinq traitements, par lignes pour les quinze groupes et par colonnes pour les trois profondeurs - effect of the five treatments by line for the 15 groups and by column for the three depths; les moyennes surmontées d'une même lettre ne sont pas significativement différentes au seuil de 5\% (test de Mann-Whitney) - the means with the same letter are not significantly different at 5\% (Mann-

Whitney's test).

\subsection{Densité de la macrofaune par groupe fonctionnel sur $0-30 \mathrm{~cm}$}

Concernant les cultures annuelles (Figure 3), c'est au niveau des cultures en ACMF que l'impact positif du non-labour sur les groupes fonctionnels (en termes de densité et de diversité) a été le plus significatif, à l'exception des géophages et des coprophages. Les parcelles en ACMF contiennent significativement plus de phytophages, de prédateurs, de rhizophages et de saprophages que les parcelles en LAMF et LAEF, et significativement moins de géophages. Il n'y a pas de différences significatives entre les modes de gestion ACMF, LAMF et LAEF pour les coprophages. Aucune différence significative n'est constatée, quel que soit le groupe fonctionnel considéré, quand on compare les modes de culture LAMF et LAEF. La quantité de fumier apportée ne semble donc pas impacter les groupes fonctionnels de la macrofaune du sol. Pour les cultures pérennes (Figure 3), les géophages sont plus nombreux dans les parcelles en CFEF alors que la densité des coprophages est plus élevée dans les parcelles en PPOF. La quantité de fumier apportée semble donc impacter les groupes fonctionnels de la macrofaune du sol.

\section{DISCUSSION}

\subsection{Effets des pratiques culturales sur la diversité et l'abondance globale de la macrofaune}

L'effet important de pratiques culturales sur la macrofaune du sol a pu être mis en évidence grâce à une comparaison de systèmes contrastés et stabilisés sur de nombreuses années. Le contraste le plus 


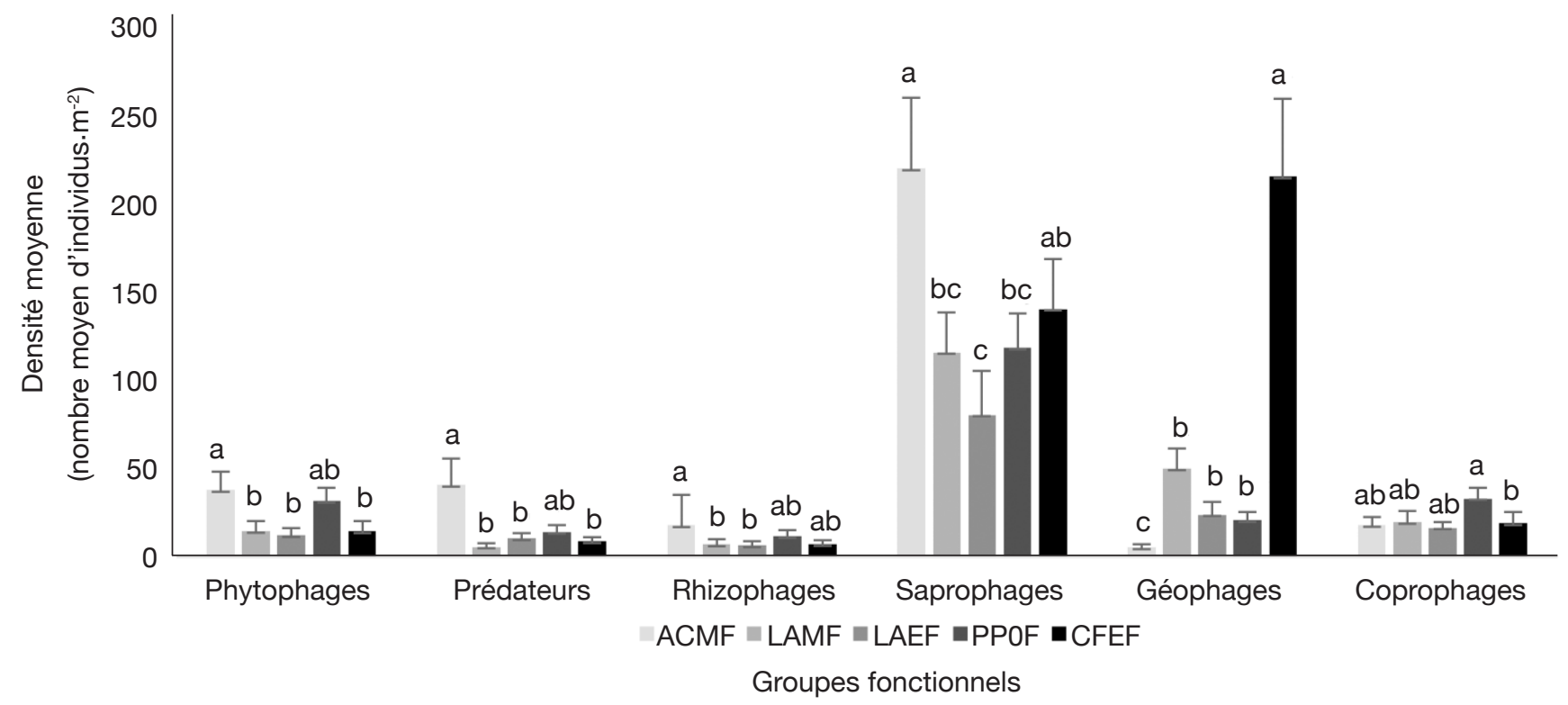

Figure 3. Densités (individus. $\left.\mathrm{m}^{-2}\right)$ moyennes de la macrofaune du sol $(0-30 \mathrm{~cm})$ pour les différents groupes fonctionnels en fonction des cinq systèmes de culture - Soil macrofauna mean densities (animals $\cdot \mathrm{m}^{-2}, 0-30 \mathrm{~cm}$ ) of the different functional groups for the five cropping systems.

Les moyennes surmontées d'une même lettre ne sont pas significativement différentes au seuil de $5 \%$ (test de Fisher et test de MannWhitney) - the means with the same letter are not significantly different at 5\% (Fisher's test and Mann-Whtiney's test) ; ACMF : cultures annuelles en agriculture de conservation sans labour et avec apport de fumier modéré - crops under conservation agriculture without tillage or permanent mulch cover and with medium manure inputs; LAMF : cultures annuelles labourées et avec apport de fumier modéré - annual crops under tillage with low manure inputs ; LAEF : cultures annuelles labourées et avec apport de fumier élevé - annual crops under tillage with high manure inputs; PP0F : pâturages permanents sans apports de fumier - permanent pastures without tillage and without manure ; CFEF : cultures fourragères pérennes avec apport de fumier élevé - permanent tilled forage crops with high manure inputs.

important a pu être établi au sein des cultures annuelles entre ACMF, LAMF et LAEF, d'une part et au sein des cultures pérennes entre PPOF et CFEF, d'autre part. Pour les cultures annuelles, la diversité et la densité de la macrofaune dans son ensemble a été plus élevée en ACMF, montrant l'effet favorable du non-travail du sol associé à la présence d'une couverture végétale permanente du sol, jouant à la fois un rôle de régulation des conditions abiotiques (température et humidité $\mathrm{du}$ sol) et d'apports constants en matières organiques (Blanchart et al., 2006 ; Ratnadass et al., 2007 ; Rabary et al., 2011). Cela confirme des études antérieures montrant l'intérêt des systèmes de culture sans labour avec semis direct sur couverture végétale pour accroître la biodiversité (densité et diversité taxonomique) de la macrofaune et dans certaines situations aussi sur la productivité des systèmes de riziculture pluviale par rapport aux systèmes conventionnels (Ratnadass et al., 2007), ou encore sur le stockage du carbone (Blanchart et al., 2007). En systèmes conventionnels avec travail du sol répété, l'hypothèse d'un impact du fumier sur la macrofaune n'a pu être confirmée par la comparaison entre LAMF et LAEF. Pour les systèmes de cultures annuelles, c'est l'effet associé du non-travail du sol et de la présence de résidus, et non la quantité de fumier, qui ont influé la quantité globale des différents groupes fonctionnels de la macrofaune. Pour les cultures pérennes, la densité en macrofaune la plus élevée a été observée au niveau des parcelles en CFEF en liaison avec un groupe fonctionnel particulier (géophages), alors qu'une plus grande diversité a été constatée au niveau des parcelles en PPOF. Dans ces premiers systèmes, la combinaison d'un travail minimal du sol associé à des apports en fumier en quantité importante semble avoir favorisé le développement de la macrofaune du sol.

\subsection{Effets des pratiques culturales sur les groupes fonctionnels de la macrofaune}

Dans notre étude, le groupe qui discrimine le plus les systèmes de culture par leur densité sont les géophages, beaucoup plus importants dans le système CFEF et en densité plus faible dans les parcelles ACMF. Les vers de terre géophages semblent donc être favorisés par 
la limitation du travail du sol en termes de fréquence et l'apport élevé en fumure organique. Le fait que ce groupe fonctionnel soit peu présent avec ACMF suggérerait un effet néfaste, soit de l'utilisation répétée des pesticides dans les parcelles gérées en ACMF pour s'affranchir d'une contrainte liée aux vers blancs (Lavelle et al., 1999), soit de l'effet de tassement du sol. En effet, à partir d'une certaine période sans labour, la population de vers de terre commence à diminuer, le sol devient trop compact. Au Brésil, Blanchart et al. (2007) ont observé au départ une augmentation de la densité et de la biomasse de vers de terre après l'arrêt du labour, suivie d'une diminution de la biomasse de vers de terre après huit ans sans labour. Cet effet néfaste est un réel problème pour le modèle de l'agriculture de conservation car les géophages jouent un rôle dans l'aération et la structuration du sol (Blanchart et al., 1999). Une présence significative plus importante des rhizophages avec ACMF par rapport à LAMF et LAEF, et cela malgré l'application de pesticides pour le mode ACMF, mériterait que l'on étudie de près les effets notamment de la moindre grande diversité culturale dans ce modèle technique. Celui-ci comprend une rotation biannuelle riz et maïs avec une seule plante de service par rapport aux pratiques conventionnelles qui offrent plus de diversité dans les successions culturales. Cet effet avait déjà été montré sur le même site par des études antérieures. Ainsi, Ratnadass et al. (2007) avaient révélé une augmentation des ravageurs du riz en agriculture de conservation après les quatre premières années de mise en place de ces systèmes, notamment par l'introduction de céréales potentiellement hôtes de ceux-ci. Sur ces systèmes, l'intérêt du traitement insecticide des semences avait été relevé. Pour éviter la prolifération de ces nuisibles des cultures, des études ont montré la possibilité d'introduire dans des successions culturales des plantes de service à effet répulsif, notamment pour cette région, comme le radis fourrager (Rabary et al., 2011). Dans notre étude, aucun effet de l'apport de fumier sur les rhizophages n'a pu être montré aussi bien en systèmes de cultures annuelles (comparaison LAMF et LAEF) qu'en systèmes de cultures pérennes (comparaison entre PPOF et CFEF). Pourtant, en Nouvelle Zélande, King (1977) avait montré que l'addition de fumier pouvait avoir un effet phagostimulant sur les vers blancs. L'ajout de matière organique sous forme de fumier pourrait accroître la décomposition de la litière grâce aux espèces saprophages obligatoires ou facultatives et favoriser ainsi la consommation de tissus végétaux vivants, stimulés par cette matière organique, par les rhizophages obligatoires. Pour les densités de phytophages, prédateurs, saprophages, c'est le mode de gestion ACMF qui a montré un effet positif et significatif par rapport aux autres modes de gestion des sols et des cultures.

\section{CONCLUSIONS}

Cette étude a permis, en étudiant des modes de gestion agricole stabilisés depuis au moins 10 années et en associant des parcelles d'agriculteurs et des sites expérimentaux, une comparaison de la biodiversité de la macrofaune dans des situations contrastées. Nous avons à la fois confirmé des résultats obtenus par certaines études précédentes et apporté les éléments nouveaux suivants :

- en systèmes de cultures annuelles conventionnels diversifiés avec labour, l'augmentation des apports de fumier ne semble pas impacter la diversité et la quantité de macrofaune du sol, qu'elle soit utile ou nuisible ;

- les pratiques conventionnelles contiendraient la pression en vers blancs nuisibles ;

- le modèle technique en agriculture de conservation maintenu depuis 15 années associant non-labour et couverture permanente du sol par des résidus de culture a favorisé la diversité de la macrofaune du sol, mais n'a pas réduit la présence de vers blancs nuisibles (malgré l'utilisation de pesticides) et ne contribue pas au développement de vers de terre géophages utiles pour le fonctionnement biologique du sol;

- dans des systèmes de cultures fourragères pérennes, associant une faible fréquence du labour et des apports importants en fumier, la présence des vers de terre a été la plus importante.

Cette approche globale de la macrofaune du sol, à la fois utile et nuisible, a montré son intérêt pour apprécier l'impact de gestions contrastées de sols et des cultures sur la biodiversité fonctionnelle du sol et sensibiliser les différents acteurs de la production agricole. Cette étude a notamment permis la publication d'un livret pour une large diffusion auprès des organisations paysannes de la région (http://www.madagascar.ird.fr/ les-activites/la-recherche/madagascar/biodiversite-etfonctionnement-des-sols-dans-les-agro-ecosystemes/ cames).

\section{Remerciements}

Cette étude a été réalisée grâce à la participation active d'agriculteurs, de techniciens de laboratoire expérimentés et de nombreux ouvriers spécialisés. Nous voulons remercier l'Agence Inter-établissements de Recherche pour le Développement (AIRD) qui a financé le projet «Impacts de l'agriculture de conservation sur la diversité de la macrofaune du sol » et les Services écosystémiques associés pour une amélioration des systèmes de culture agricole et de la qualité de vie des paysans sur les Hautes-Terres de Madagascar (CAMES) et l'ensemble de ce travail. 


\section{Bibliographie}

Alvarez S. et al., 2012. Whole-farm nitrogen cycling and intensification of crop-livestock systems in the highlands of Madagascar: an application of network analysis. Agric. Syst., 126, 25-37.

Anderson J.M. \& Ingram J., eds, 1993. Tropical soil biology and fertility. A handbook of methods. $2^{\text {nd }}$ ed. Wallingford, UK: CAB International.

Avelino J. et al., 2012. Incorporating plant species diversity in cropping systems for pest and disease risk management. Forum for Agricultural Risk Management in Development, http://www.agriskmanagementforum.org/, (29/09/16).

Bartz M.L.C., Pasini A. \& Brown G.G., 2013. Earthworms as soil quality indicators in Brazilian no tillage systems. Appl. Soil Ecol., 69, 39-48.

Blanchart E. et al., 1999. Effects of earthworms on soil structure and physical properties. In: Lavelle P., Brussaard L. \& Hendrix P., eds. Earthworms management in tropical agroecosystems. New York, NY, USA: CABI, 149-172.

Blanchart E. et al., 2006. Long-term effect of a legum cover crop (Mucuna pruriens var. utilis) on the communities of soil macrofauna and nematofauna, under maize cultivation, in southern Benin. Eur. J. Soil Biol., 42, 136-144.

Blanchart E. et al., 2007. Effect of direct seeding mulch-based systems on soil carbon storage and macrofauna in central Brazil. Agric. Conspectus Scientificus, 72, 81-87.

Csuzdi C., Razafindrakoto M. \& Blanchart E., 2012. New and little known earthworm species from Central Madagascar (Oligochaeta: Kynotidae). Zootaxa, 3578, 36-42.

Dechambre R.P., 1986. Insectes Coléoptères Dynastidae. Faune de Madagascar 65. Paris: Museum National d'Histoire Naturelle.

Djigal D. et al., 2011. Mulch type affects soil biological functioning and crop yields of conservation agriculture systems in a long-term experiment in Madagascar. Soil Tillage Res., 118, 11-21.

Dusserre J. et al., 2012. Upland rice production under conservation agriculture cropping systems in cold conditions of tropical highlands. Field Crops Res., 138, 33-41.

King P.D., 1977. Effect of plant species and organic matter on feeding behaviour and weight gain of larval black beetle, Heteronychus arator (Coleoptera: Scarabaeidae). New Zealand J. Zool., 4, 445-448.

Lacroix M., 1989. Insectes Coléoptères Melolonthidae. Paris: Museum national d'Histoire naturelle. Faune de Madagascar 73(1).

Lacroix M., 1993. Insectes Coléoptères Melolonthidae. Paris: Museum national d'Histoire naturelle. Faune de Madagascar 73(2).

Lacroix M., 1997. Insectes Coléoptères Hopliidae. Paris : Museum national d'Histoire naturelle. Faune de Madagascar 88(1).
Lacroix M., 1998. Insectes Coléoptères Hopliidae. Paris : Museum national d'Histoire naturelle. Faune de Madagascar 88(2).

Lavelle P., Brussaard L. \& Hendrix P., eds, 1999. Earthworm management in tropical agroecosystems. Wallingford, UK: CABI Publishing, 149-172.

Lavelle P. et al., 2001. SOM management in the tropics: why feeding the soil macrofauna? Nutr. Cycl. Agroecosyst., 61, 53-61.

Lavelle P. et al., 2006. Soil invertebrates and ecosystem services. Eur. J. Soil Biol., 42, 3-15.

Rabary B. et al., 2011. White grubs, Scarabaeidae larvae (Insecta, Coleoptera) control by plants in conservation agriculture: effects on macrofauna diversity. In: Proceedings of the $5^{\text {th }}$ World Congress of Conservation Agriculture, 26-29 September 2011, Brisbane, Australia.

Raboin L.M. et al., 2013. Création variétale pour la riziculture pluviale d'altitude à Madagascar : bilan de 25 années de sélection. Cah. Agric., 22, 1-9.

Randriamanantsoa R.et al., 2010.Les larves des Scarabaeoidea (Insecta, Coleoptera) en riziculture pluviale des régions de haute et moyenne altitudes du Centre de Madagascar. Zoosystema, 32, 19-72.

Ratnadass A. et al., 2007. Impacts d'un système de culture à base de riz pluvial et de semis direct sur couverture végétale $(\mathrm{SCV})$ sur la macrofaune du sol à Madagascar, avec référence particulière aux effets sur la production du riz. Terre Malgache, 26, 39-41.

Ratnadass A. et al., 2013. Interaction entre le système de culture et le statut (ravageur ou auxiliaire) des vers blancs (Coleoptera: Scarabeoidea) sur le riz pluvial. Cah. Agric., 22, 1-10.

Razafindrakoto M., Csuzdi C., Rakotofiringa S. \& Blanchart E., 2010. New records of earthworms (Oligochaeta) from Madagascar. Opusc. Zool., 41, 231236.

Sester M. et al., 2015. Évaluer la durabilité de systèmes de culture en agriculture de conservation à Madagascar (région du lac Alaotra) avec MASC-Mada. Cah. Agric., 24, 123-133.

Tonneau J.P., Sabourin E., Marçal da Silveira L. \& Sidersky P., 2002. Modélisation des flux de biomasse : une approche de la fertilité dans l'Agreste de la Paraíba (Brésil). Cah. Agric., 11, 127-136.

Turbé A. et al., 2010. Soil biodiversity: functions, threats and tools for policy makers. Report for European Commission (DG Environment). Paris: Bio Intelligence Service, IRD ; Wageningen, The Netherlands : NIOO. 